\title{
Numerical modeling method for the dispersion characteristics of single-mode and multimode weakly-guiding optical fibers with arbitrary radial refractive index profiles
}

\author{
Raushan Mussina ${ }^{* a}$, David R. Selviah ${ }^{\mathrm{a}}$, F. Aníbal Fernández ${ }^{\mathrm{a}}$, \\ Anton G. Tijhuis ${ }^{\mathrm{b}}$, and Bastiaan P. de Hon ${ }^{\mathrm{b}}$ \\ ${ }^{a}$ Department of Electronic and Electrical Engineering, University College London, UCL, London, \\ WC1E 7JE, UK; ${ }^{b}$ Faculty of Electrical Engineering, University of Technology, $5600 \mathrm{MB}$ \\ Eindhoven, the Netherlands
}

\begin{abstract}
Accurate, reliable and fast numerical modeling methods are required to design the optimum radial refractive index profile for single and multimode fibers to give specific dispersion characteristics prior to or even obviating costly experimental work. Such profiles include graded index and multiple concentric cladding layers. In this paper, a new numerical method is introduced which enables the derivatives of the propagation coefficient to be calculated analytically up to the third order of a single mode or multimode weakly guiding optical fiber with an arbitrary radial refractive index profile. These quantities are required to determine the group delay, $\tau_{\mathrm{g}}$, chromatic dispersion, $D$, and dispersion slope of the fiber. The expansion of the modal fields in terms of Laguerre-Gauss polynomials in the Galerkin method offers certain benefits. In particular, due to simplicity of the basis functions it is possible to carry out further analytical work on the results such as repeated differentiation of the matrix equation resulting from the Galerkin method to define up to the third-order derivatives of the propagation coefficients with respect to wavelength. This avoids approximation errors inherent in numerical differentiation, giving better accuracy and, at the same time, significantly reduces the computation time. A computer program was developed to demonstrate the proposed method for single and multimode fibers with radially arbitrary refractive index profiles. The paper provides simulation results to validate the approach.
\end{abstract}

Keywords: dispersion, dispersion slope, single-mode fibers, few-mode fibers, step-index fibers, double-clad fibers, triple-clad fibers, graded index profile, analytical dispersion evaluation, Galerkin method

\section{INTRODUCTION}

By suitably tailoring the refractive index profile parameters of single and multimode fibers, the dispersion spectrum can be modified so that a desired shape of dispersion curve can be achieved. Single-mode fibers play an important role in current high-capacity data transmission while multimode fibers (or few-mode fibers to be exact) are considered for space-division multiplexing so as to address the growing demand for broadband communications and attracting increasing attention [1-3].

When it comes to optimizing refractive index profiles in the design of single or few-mode optical fibers, the minimization of the cost function heavily depends on the accuracy and speed of the numerical evaluation of fiber dispersion characteristics. Consequently, accurate and fast numerical modeling methods are required to give specific dispersion characteristics without the need for costly and time consuming experimental fabrication and measurements.

In this paper, we introduce a new numerical method to analytically determine the first, second and third derivatives of the propagation coefficient required to calculate the group delay, $\tau_{\mathrm{g}}$, chromatic dispersion, $D$, and dispersion slope of single and few-mode weakly guiding optical fibers having arbitrary radial refractive index profiles. This approach employs the Galerkin method as a mode solver for the scalar wave equation [4] and applies repeated analytical differentiation to the matrix equation resulting from the Galerkin method to define up to the third-order derivatives of the

*r.mussina@ee.ucl.ac.uk; phone +44(0) 20 7679 4157; fax +44 (0) 207388 9325; www.ee.ucl.ac.uk/

Physics and Simulation of Optoelectronic Devices XXI, edited by Bernd Witzigmann, Marek Osinski,

Fritz Henneberger, Yasuhiko Arakawa, Proc. of SPIE Vol. 8619, 86191R · @ 2013 SPIE

CCC code: $0277-786 X / 13 / \$ 18 \cdot$ doi: $10.1117 / 12.2002804$ 
propagation coefficient with respect to wavelength. This avoids the approximation errors inherent in numerical differentiation, giving better accuracy and, at the same time, significantly reducing the computation time.

So far, most research has been focused on approximate methods [5-10] to calculate dispersion, and, except from the fullwave approach of [10] has not included the calculation of dispersion slope. However, for the design of optical fibers, knowledge of the dispersion slope is now becoming as important as the knowledge of the dispersion coefficient. When numerical differentiation is used for the calculation of the propagation coefficient derivatives, then, in addition to introducing approximation errors, the overall method slows down, e.g, the eigenvalue problem is usually solved five times for the determination of dispersion and dispersion slope. An analytical approach for the evaluation of the first and second derivatives of the propagation coefficient for photonic crystal fibers was recently reported in [11] which combines an iterative Fourier technique to compute the propagation coefficient at any fixed wavelength and an analytical procedure to calculate chromatic dispersion.

The proposed method in this paper takes full advantage of the Galerkin method: simplicity of the Laguerre-Gauss basis functions allows repeated differentiation of the symmetric matrix equation resulting from it up to the third order, thus giving the combination of an accurate and very fast mode solver and analytical differentiation. A computer program was developed to demonstrate the proposed method for single and few-mode fibers with radially arbitrary refractive index profiles including graded index, step index and multi-clad fibers. The wavelength dependence of the refractive indices of the core and cladding materials are incorporated in the model so that the program calculates the total chromatic dispersion. The paper provides simulation results to validate the new numerical method.

\section{THEORY}

\subsection{Optical fiber dispersion characteristics: group delay, dispersion and dispersion slope}

The wavelength $(\lambda)$ or frequency $(\omega)$ dependent nature of dispersion is described through the group delay time, $\tau_{\mathrm{g}}$, per unit length. This group slowness is the reciprocal of the group velocity, $v_{\mathrm{g}}$, at which a pulse propagates through the fiber [12]:

$$
\tau_{\mathrm{g}}[s / k m]=\frac{1}{v_{\mathrm{g}}}=\frac{d \beta}{d \omega}
$$

where $\beta$ is the propagation coefficient of the guided mode.

The dispersion, $D$, per unit length is defined as [12]

$$
D[p s /(n m \cdot k m)]=\frac{d \tau_{\mathrm{g}}}{d \lambda}=-\frac{\lambda}{c} \frac{d^{2}}{d \lambda^{2}}\left(\frac{\beta}{k_{0}}\right)=-\frac{2 \pi c}{\lambda^{2}} \frac{d^{2} \beta}{d \omega^{2}},
$$

where $c$ is the speed of light in a vacuum and $k_{0}$ is the free-space wavenumber.

The dispersion slope is the derivative of the dispersion, $D$, with respect to wavelength:

$$
D S\left[p s /\left(n m^{2} \cdot k m\right)\right]=\frac{d D}{d \lambda},
$$

and thus proportional to the third-order derivative of the propagation coefficient.

\subsection{Weakly guiding optical fiber model and refractive index profile}

The application of the Galerkin method with Laguerre-Gauss basis functions to a weakly guiding optical fiber model is described in full detail in [4] by Meunier, et al. It transforms the wave equation for the transverse electromagnetic fields

$$
\left[\nabla_{t}^{2}+k_{0}^{2} n^{2}(r)\right] \psi(r, \theta)=\beta^{2} \psi(r, \theta)
$$


where $n(r)$ is the refractive index profile of the fiber and $\nabla_{t}^{2}$ is the transverse part of the Laplacian operator, into a matrix eigenvalue problem

$$
\mathbf{A c}=\beta^{2} \mathbf{c}
$$

where $\mathbf{A}$ is a real symmetric matrix, which has purely discrete real eigenvalues which correspond to the propagation coefficients, $\beta$, and the components of the eigenvectors represent the expansion coefficients of the modes in terms of basis functions. Due to circular symmetry, the refractive index profile, $n(r)$, is assumed to depend on only the radial component and is defined by

$$
n^{2}(r)= \begin{cases}n_{\mathrm{co}}^{2}\left[1-2 \Delta f\left(\frac{r}{r_{0}}\right)\right], & r \leq r_{0} \\ n_{\mathrm{cl}}^{2}=n_{\mathrm{co}}^{2}[1-2 \Delta f(1)], & r>r_{0}\end{cases}
$$

where $r_{0}$ is the core radius, $n_{\mathrm{co}}$ and $n_{\mathrm{cl}}$ are the refractive indices at the core center and the cladding respectively. The relative refractive index is $\Delta=\left(n_{\mathrm{co}}^{2}-n_{\mathrm{cl}}^{2}\right) /\left(2 n_{\mathrm{co}}^{2}\right)$, and the profile function $f\left(r / r_{0}\right)$ is normalized so that $f(0)=0$ and $f(1)=1$. Figure 1 (a) shows the reference infinitely extended parabolic profile for which the Laguerre-Gauss polynomials are the eigensolutions. The schematic refractive index profiles of the single or few-mode fibers under consideration are given in Figures 1(b), (c) and (d): (b) step-index, (c) truncated parabolic and (d) arbitrary refractive index profiles.
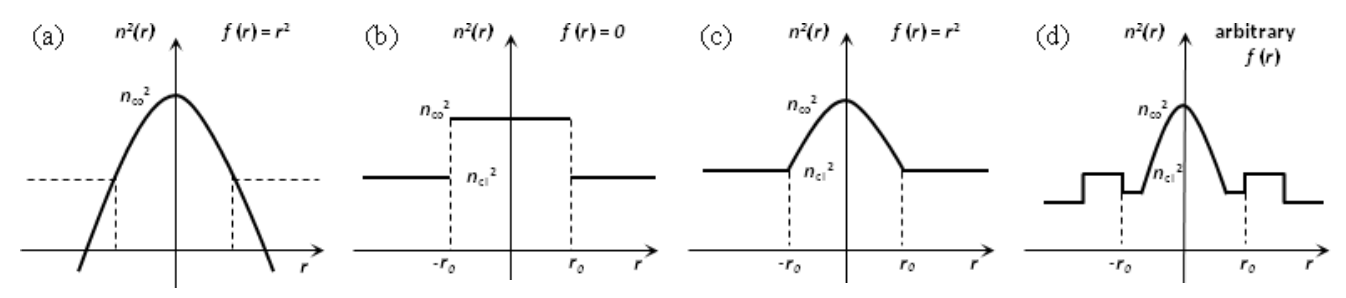

Figure 1. Schematic refractive index profiles: (a) infinitely extended parabolic profile; (b) step-index profile; (c) truncated parabolic profile; (d) arbitrary profile

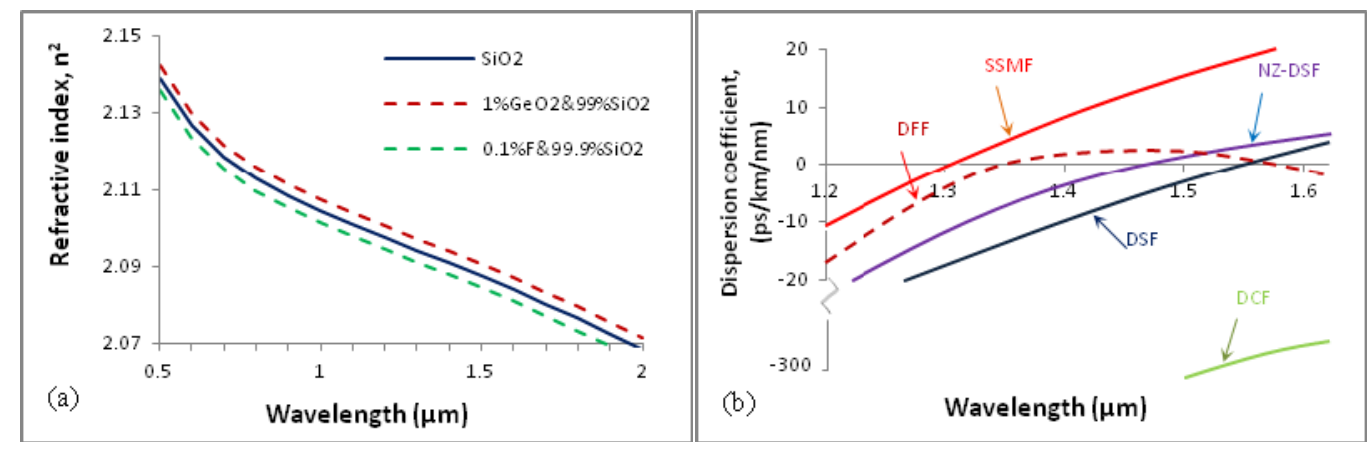

Figure 2. (a) Refractive index plotted as a function of wavelength for pure and doped silica; (b) Typical dispersion spectrum for different types of single-mode fibers [14]: standard single-mode fiber (SSMF), dispersion-shifted fiber (DSF), non-zero dispersion-shifted fiber (NZ-DSF), dispersion-flattened fiber (DFF) and dispersion-compensating fiber (DCF)

The Sellmeier equations [13] are used to take into account the wavelength dependence of the refractive indices of the core and cladding materials:

$$
n^{2}(\lambda)=1+\sum_{i=1}^{3} \frac{\alpha_{i} \lambda^{2}}{\lambda^{2}-l_{i}^{2}}
$$


where $\alpha_{i}$ and $l_{i}$ are material specific Sellmeier parameters given in [13] for various glass composites. With respect to a reference composite doping level, $d_{0}$, the refractive index for a doping level $d$ is defined by [5]

$$
n(\lambda, d)=\left[n\left(\lambda, d_{0}\right)-n(\lambda, 0)\right] \frac{d}{d_{0}}+n(\lambda, 0)
$$

where $n\left(\lambda, d_{0}\right)$ and $n(\lambda, 0)$ are the respective refractive indices at the reference doping level and of pure silica, $\mathrm{SiO}_{2}$.

Figure 2(a) depicts the wavelength dependent behavior of the refractive index for pure and doped silica; it is worth noting that the use of germania, $\mathrm{GeO}_{2}$, as a dopant increases the refractive index, while fluorine, F, decreases it.

By tailoring the refractive index profile parameters a desired shape of the dispersion graph can be obtained. Typical dispersion plots for various single-mode fiber types are shown in Figure 2(b). These include standard single-mode fiber (SSMF), dispersion-shifted fiber (DSF), non-zero dispersion-shifted fiber (NZ-DSF), dispersion-flattened fiber (DFF) and dispersion-compensating fiber (DCF) [14].

\subsection{The proposed method for the calculation of dispersion characteristics}

Equations (1)-(3) show that the first, second and third-order derivatives of the propagation coefficient with respect to wavelength or frequency are needed to evaluate the dispersion characteristics of optical fibers. In order to calculate the derivatives of the propagation coefficient, we perform analytical differentiation of the matrix equation (5) with respect to frequency, $\omega$, along with the condition for the normality of the eigenvector $\mathbf{c}$

$$
\|\mathbf{c}\|=1 \quad \text { or } \quad \mathbf{c}^{\mathrm{T}} \mathbf{c}=1
$$

where $\mathbf{c}^{\mathrm{T}}$ is the transpose of $\mathbf{c}$.

All of the terms in equation (5), $\mathbf{A}, \beta^{2}$ and $\mathbf{c}$, depend on the angular frequency, $\omega$. Thus, differentiation of equations (5) and (9) with respect to $\omega$ gives

$$
\frac{d \mathbf{A}}{d \omega} \mathbf{c}+\mathbf{A} \frac{d \mathbf{c}}{d \omega}=\frac{d \beta^{2}}{d \omega} \mathbf{c}+\beta^{2} \frac{d \mathbf{c}}{d \omega}
$$

and

$$
\frac{d \mathbf{c}^{\mathrm{T}}}{d \omega} \mathbf{c}+\mathbf{c}^{\mathrm{T}} \frac{d \mathbf{c}}{d \omega}=0 \quad \Rightarrow \quad \mathbf{c}^{\mathrm{T}} \frac{d \mathbf{c}}{d \omega}=0 .
$$

Multiplication of equation (10) by $\mathbf{c}^{\mathrm{T}}$ on the left gives

$$
\mathbf{c}^{\mathrm{T}} \frac{d \mathbf{A}}{d \omega} \mathbf{c}+\mathbf{c}^{\mathrm{T}}\left(\mathbf{A}-\beta^{2} \mathbf{I}\right) \frac{d \mathbf{c}}{d \omega}=\frac{d \beta^{2}}{d \omega} \mathbf{c}^{\mathrm{T}} \mathbf{c},
$$

which, due to (9) and $\mathbf{c}^{\mathrm{T}}\left(\mathbf{A}-\beta^{2} \mathbf{I}\right)=0$ (as $\mathbf{A}$ is a symmetric matrix), gives

$$
\frac{d \beta^{2}}{d \omega}=\mathbf{c}^{\mathrm{T}} \frac{d \mathbf{A}}{d \omega} \mathbf{c},
$$

or equivalently,

$$
\frac{d \beta}{d \omega}=\frac{1}{2 \beta} \mathbf{c}^{\mathrm{T}} \frac{d \mathbf{A}}{d \omega} \mathbf{c} .
$$

This is the first derivative of the propagation coefficient. To calculate it we need $d \mathbf{A} / d \omega$, the derivative of matrix $\mathbf{A}$, which we do by differentiating the terms of $\mathbf{A}$ analytically.

Similarly, by repeatedly differentiating equation (5), the second and third derivatives of $\beta$ are evaluated according to

$$
\frac{d^{2} \beta}{d \omega^{2}}=\frac{1}{2 \beta}\left[\frac{d^{2} \beta^{2}}{d \omega^{2}}-2\left(\frac{d \beta}{d \omega}\right)^{2}\right],
$$


where

$$
\frac{d^{2} \beta^{2}}{d \omega^{2}}=\mathbf{c}^{\mathrm{T}}\left(\frac{d^{2} \mathbf{A}}{d \omega^{2}}\right) \mathbf{c}+2 \mathbf{c}^{\mathrm{T}} \frac{d \mathbf{A}}{d \omega} \frac{d \mathbf{c}}{d \omega},
$$

and

$$
\frac{d^{3} \beta}{d \omega^{3}}=\frac{1}{2 \beta}\left[\frac{d^{3} \beta^{2}}{d \omega^{3}}-6 \frac{d \beta}{d \omega} \frac{d^{2} \beta}{d \omega^{2}}\right]
$$

with

$$
\frac{d^{3} \beta^{2}}{d \omega^{3}}=\mathbf{c}^{\mathrm{T}}\left(\frac{d^{3} \mathbf{A}}{d \omega^{3}}\right) \mathbf{c}+3 \mathbf{c}^{\mathrm{T}}\left(\frac{d^{2} \mathbf{A}}{d \omega^{2}}\right) \frac{d \mathbf{c}}{d \omega}+3 \mathbf{c}^{\mathrm{T}}\left(\frac{d \mathbf{A}}{d \omega}-\frac{d \beta^{2}}{d \omega} \mathbf{I}\right) \frac{d^{2} \mathbf{c}}{d \omega^{2}} .
$$

From equations (16) and (18) it can be seen that the second and third derivatives of $\beta$ require the determination of the first and second derivatives of the eigenvector $\mathbf{c}$. For the calculation of eigenvector derivatives $d \mathbf{c} / d \omega$ and $d^{2} \mathbf{c} / d \omega^{2}$, the method proposed in [15] was used. The second and third derivatives of matrix $\mathbf{A}$ are evaluated analytically.

\section{NUMERICAL RESULTS}

\subsection{Group delay, dispersion and dispersion slope graphs}

Table 1. Core and cladding parameters for exemplary single-, double-, and triple-clad fibers in Figure 3

\begin{tabular}{|llll|}
\hline \multicolumn{4}{c|}{ FIBER PARAMETERS } \\
\hline \hline & Core material & Core and cladding radii & $\begin{array}{l}\text { Dopants used in cladding } \\
\text { with the doping levels }\end{array}$ \\
\hline \hline Single-clad fiber & pure $\mathrm{SiO}_{2}$ & $3.5 \mu \mathrm{m}$ & Fluorine, $1.782 \%$ \\
\hline Double-clad fiber & pure $\mathrm{SiO}_{2}$ & $4.2 \mu \mathrm{m}, 5.2 \mu \mathrm{m}$ & Fluorine, $4.5 \%, 1.08 \%$ \\
\hline Triple-clad fiber & pure $\mathrm{SiO}_{2}$ & $\begin{array}{l}4.2 \mu \mathrm{m}, 8.25 \mu \mathrm{m}, \\
\text { and } 15 \mu \mathrm{m}\end{array}$ & $\begin{array}{l}\text { Fluorine, } 1.782 \%, \\
0.509 \%, \text { and } 1.131 \%\end{array}$ \\
\hline
\end{tabular}

Figure 3 compares the graphs of the group delay, total dispersion coefficient and dispersion slope for particular examples of single-clad, double-clad and triple-clad fibers. The parameters of the fiber designs are given in Table 1 showing cladding layers with different concentrations of fluorine doping which are typical of those used in the fiber manufacturing industry. The tpiple-clad fiber was designed in [16]. The graphs in Figure 3 clearly illustrate the relationship between the three fiber dispersion characteristics: dispersion is proportional to the derivative of the group delay with respect to wavelength, and dispersion slope is in turn proportional to the derivative of dispersion as a function of wavelength. In Figure 3(a), the group delay for a single-clad fiber (the blue curve) has a minimum point which corresponds to the only zero crossing of this fiber's dispersion graph displayed in Figure 3(b). The dispersion graph is monotonically rising, and consequently its dispersion slope is a positive function shown in Figure 3(c). The cases of the double- and triple-clad fibers are illustrated by the green and red curves respectively. In these examples, the dispersion graphs have two or more zeros. Hence, the corresponding group delay graphs have two or more local extrema, and the dispersion slope graphs have zero crossing points at the extrema of the dispersion. 

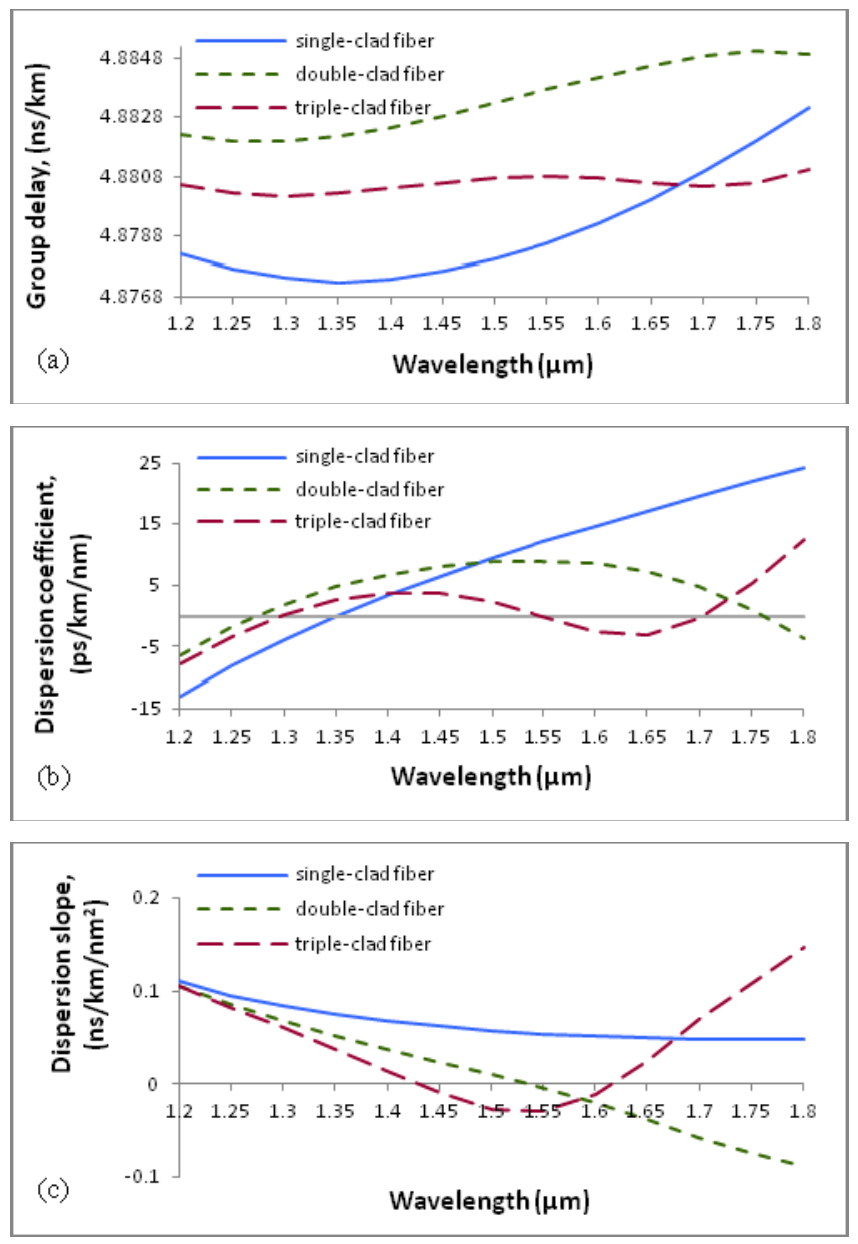

Figure 3. Comparative dispersion characteristics of exemplary single-, double- and triple-clad fibers: (a) group delay; (b) chromatic dispersion; (c) dispersion slope

\subsection{Comparison of simulation results to experimental data}

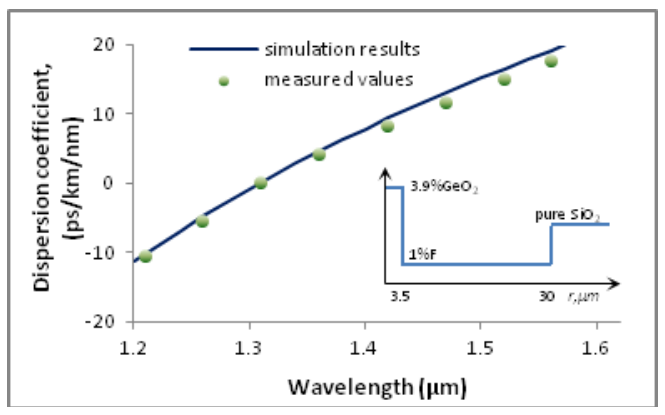

Figure 4. Dispersion graph for the depressed cladding fiber from [17] compared to measured values

Next, let us consider an example that compares simulation results with experimental data given in [17] for a depressed cladding fiber with a 3.9\% Ge-doped plasma induced chemical vapor deposition (PCVD) silica core region surrounded by a $1 \%$ fluorine-doped PCVD silica region that forms the depressed cladding and pure silica outer cladding (the radii are 3.5 and $30 \mu \mathrm{m})$. The Sellmeier parameters for germania and fluorine doped PCVD silica are defined according to 
expressions given in [17]. A good agreement was established between the simulated and experimental data as can be seen from Figure 4.

\subsection{Tabulated dispersion characteristics values}

The modeled values of the effective propagation coefficient, group delay, dispersion and dispersion slope for a truncated parabolic profile fiber are tabulated in Table 2 for various matrix sizes. The computation times for the determination of the propagation coefficient, group delay, dispersion and dispersion slope for $N=25,35$ and 45 was $0.059 \mathrm{~s}, 0.195 \mathrm{~s}$ and 0.4 s respectively (Intel Pentium(R) CPU $2 \mathrm{GHz}$ ). For comparison: the time required to calculate a single dispersion value at a fixed wavelength in [11] is about $5 \mathrm{~min}$.

Table 2. Dispersion characteristics quantities for a single-mode fiber with Ge-doped core and pure $\mathrm{SiO}_{2}$ cladding

Truncated parabolic profile

core: $13.5 \% \mathrm{GeO}_{2}, 86.5 \% \mathrm{SiO}_{2}$, cladding: pure $\mathrm{SiO}_{2}$, core radius: $2.5 \mu \mathrm{m}, \lambda=1.75 \mu \mathrm{m}$

\begin{tabular}{ccccc}
\hline \hline Matrix size, $\boldsymbol{N}$ & $\begin{array}{c}\text { Effective } \\
\text { propagation } \\
\text { coefficient, } \boldsymbol{\beta} / \boldsymbol{k}_{\boldsymbol{0}}\end{array}$ & $\begin{array}{c}\text { Group delay, } \boldsymbol{\tau}_{\boldsymbol{g}} \\
(\mathbf{n s} / \mathbf{k m})\end{array}$ & $\begin{array}{c}\text { Dispersion, } \boldsymbol{D} \\
(\mathbf{p s} / \boldsymbol{n m} / \mathbf{k m})\end{array}$ & $\begin{array}{c}\text { Dispersion slope, } \\
\boldsymbol{D S}\left(\boldsymbol{n s} / \boldsymbol{n m} \boldsymbol{m}^{2} / \mathbf{k m}\right)\end{array}$ \\
\hline \hline 5 & 1.44627916 & 4.93459345 & 7.54688736 & 0.05960322 \\
15 & 1.44635525 & 4.93317160 & 2.80932819 & 0.05017661 \\
25 & 1.44635615 & 4.93313637 & 2.54241550 & 0.04803728 \\
35 & 1.44635620 & 4.93313384 & 2.51601666 & 0.04801827 \\
45 & 1.44635631 & 4.93313348 & 2.50867604 & 0.04797553 \\
\hline \hline
\end{tabular}

\section{CONCLUSION}

We have described a method for the calculation of the group delay, dispersion and dispersion slope parameters of single and few-mode weakly guiding optical fibers with radially arbitrary refractive index profiles. The method relies on analytical differentiation of the matrix equation for the determination of the first, second and third order derivatives of the propagation coefficient. The numerical results show that the method yields fast and accurate results.

\section{ACKNOWLEDGEMENTS}

Raushan Mussina gratefully acknowledges the support of the Republic of Kazakhstan via the Bolashak Scholarship Programme and the European Union via the Erasmus Mundus External Cooperation Window Mobility Grant Programme.

\section{REFERENCES}

[1] Li A., Al Amin A., Chen X., and Shieh W., “ Reception of mode and polarization multiplexed 107-Gb/s COOFDM signal over a two-mode fiber" Optical Fiber Communication Conference (OFC), PDPB8 (2011).

[2] Salsi M., Koebele C., Sperti D., Tran P., Brindel P., Mardoyan H., Bigo S., Boutin A., Verluise F., Sillard P., Bigot-Astruc M., Provost L., Cerou F., and Charlet G., "Transmission at 2x100Gb/s, over two modes of 40kmlong prototype few-mode fiber, using LCOS based mode multiplexer and demultiplexer," Optical Fiber Communication Conference (OFC), PDPB9 (2011).

[3] Ryf R., Randel S., Gnauck A. H., Bolle C., Essiambre R., Winzer P., Peckham D. W., McCurdy A., and Lingle R., "Space-division multiplexing over $10 \mathrm{~km}$ of three-mode fiber using coherent $6 \times 6$ MIMO processing," Optical Fiber Communication Conference (OFC), PDPB10 (2011).

[4] Meunier J. P., Pigeon J., and Massot J. N., "A general approach to the numerical determination of modal propagation constants and field distributions of optical fibres," Opt. Quant. Electron. 13(1), 71-83 (1981). 
[5] Sharma A. and Banerjee S., "Chromatic dispersion in single mode fibers with arbitrary index profiles: a simple method for exact numerical evaluation," J. Lightwave Technol. 7(12), 1919-1923 (1989).

[6] Kim J. and Kim D. Y., "An efficient dispersion calculation method for axially symmetric optical fibers," Fiber Integrated Opt. 21(1), 13-29 (2002).

[7] Li Q. Y., "Propagation characteristics of single-mode optical fibers with arbitrary refractive-index profile: the finite quadratic element approach," J. Lightwave Technol. 9(1), 22-26 (1991).

[8] Boucouvalas A. C. and Qian X., "Mode dispersion and delay characteristics of optical waveguides using equivalent TL circuits," IEEE J. Quantum Electron., 41(7), 951-957 (2005).

[9] Lin H. Y., Wu R. B., and Chang H. C., "An efficient algorithm for determining the dispersion characteristics of single-mode optical fibers," J. Lightwave Technol. 10(6), 705-711 (1992).

[10] Smink, R.W., de Hon, B.P., Bingle, M., Mussina, R., and Tijhuis, A.G., "Refractive index profile optimisation for the design of optical fibres," Opt. Quant. Electron., 40(11-12), 837-852 (2008).

[11] Silvestre E., Pinheiro-Ortega T., Andrres P., Miret J. J., and Ortigosa-Blanch A., "Analytical evaluation of chromatic dispersion in photonic crystal fibers," Opt. Lett. 30(5), 453-455 (2005).

[12] Snyder A. W. and Love J. D., [Optical waveguide theory], Chapman and Hall, London, (1983).

[13]Fleming J. W., "Material dispersion in lightguide glasses," Electron. Lett. 14(11), 326-328 (1978).

[14] Senior J. M., [Optical fiber communications: principles and practice], third ed., Pearson Education Limited, (2009).

[15] Wu B. S., Xu Z. H., and Li Z. G., "A note on computing eigenvector derivatives with distinct and repeated eigenvalues," Commun. Numer. Meth. En. 23(3), 241-251 (2007).

[16]Etzkorn H., "Low-dispersion single-mode silica fibre with undoped core and three F-doped claddings," Electron. Lett. 20(10), 423-424 (1984).

[17] Hermann W. and Wiechert D. U., "Refractive-index of doped and undoped PCVD bulk silica," Mater. Res. Bull. 24(9), 1083-1097 (1989). 